\title{
Importancia del medio ambiente en el recién nacido bajo peso
}

DRES.: J. ALVEAR *, L. ALVAREZ *, L. COUSINO * Y F. MONCKEBERG *.

Hay numerosos estudios que demuestran una significativa relación entre bajo peso de nacimiento, con tasas elevadas de mortalidad natal $(1,2$, 3 ,4) o daños neurológicos $\mathrm{y} / \mathrm{o}$ retraso mental (6). Se ha establecido que el peso de nacimiento en sujetos con igual edad gestacional se relaciona con la dieta que recibe la madre $(7,8)$ transporte placentario adecuado y aprovechamiento normal de los nutrientes por parte del feto (9).

Dentro de un mismo grupo étnico el mejoramiento de las condiciones sociales, incluyendo nutrición, generalmente se refleja en un incremento de los pesos de nacimiento (3). Desgraciadamente también ocurre lo inverso, como lo han demostrado estudios hechos en tiempo de guerra (10) .

Esto se observa a nivel animal en los que la reducción de la calidad y/o cantidad de las dietas de las madres reduce el número y peso de los recién nacidos, que mantienen su retraso de crecimiento aún cuando se les coloque en condiciones ideales después de nacidos, si los comparamos con hijos de madres bien alimentadas (11).

Se ha establecido claramente que la desnutrición compromete el crecimiento de órganos, tejidos especialmente de aquellos que en ese momento no han alcanzado su total desarrollo, por lo que las manifestaciones varían según la etapa de la vida en que se produzca. $(12,13,14,15,16$, 17). Si a esto sumamos un medio ambiente inadecuado o pobre en estímulos, colocamos al sujeto en clara desventaja respecto a sus semejantes que crecieron en condiciones adecuadas (14).

En Chile el $10 \%$ a $15 \%$ de los recién nacidos nacen con un peso inferior a 2.500 gr. (18). Pretendemos estudiar en que forma este bajo peso de nacimiento se refleja en el posterior desarrollo y crecimiento de estos niños.

\footnotetext{
* Departamento de Nutriclón y Tecnología de los Alimentos. Universidad de Chile. Sede Sur.
}

Material y METOdo. Este es un estudio retrospectivo y para esto se eligió como área de estudios la maternidad del Hospital del Salvador por abarcar uno de los sectores más populares del Gran Santiago y por la representatividad social del grupo que la habita.

Se revisaron las fichas de los 11.898 nacimientos ocurridos durante 1970. Se escogió este año por considerar que en la actualidad a los 3 años de edad los niños ya presentan influencias del medio ambiente.

Del total de niños, 1.355 nacieron con un peso inferior a 2.500 gr. $(11,4 \%)$; de este grupo se seleccionó aquellos nacidos de término considerándose como tales los nacidos con 38 a 42 semanas de edad gestacional, obteniéndose 183 casos.

Se seleccionó una muestra de estos niños obteniéndose 47 casos, 20 hombres y 25 mujeres.

La muestra control se obtuvo de los 10.269 niños nacidos de embarazos de término con peso superior a $2.500 \mathrm{gr}$. seleccionándose el $0,2 \%$ de ellos constituidos por 9 niños y 9 niñas (20).

\section{Variables medidas:}

Se recolectaron tres tipos de datos índices a) Nivel socioeconómico del grupo familiar; b) Crecimiento físico y evaluación nutricional y c) Evaluación del desarrollo psicomotriz del niño en estudio.

\section{a) Nivel socioeconómico familiar:}

Se realizó una encuesta socioeconómica en el domicilio (19) en la que además se investigó retrospectivamente estado de salud de la madre durante el embarazo, antecedentes del niño y hábitos alimentarios del grupo familiar. 
b) Crecimiento del niño en estudio:

Peso, talla de pie y sentado, circunferencia de cráneo y edad ósea por medio de radiografía de carpo. Exámenes sanguíneos orientados a la búsqueda de deficiencias alimentarias como hemoglobina, hematocritos, proteinemia, índice A/G, fosfemia, calcemia y examen parasitológico seriado de deposiciones como índice de saneamiento ambiental.

\section{c) Evaluación psicométrica del niño:}

Se utilizó el test de Arnold Gessel y Ozeretzqui (21) pasado por personal adiestrado y controlado por psicólogo.

Resultados. Las características socioeconómicos del grupo familiar de ambos grupos estudiados fueron similares y no se observaron diferencias significativas, en relación a ninguno de los ítems estudiados como tamaño familiar, escolaridad de los padres, ingresos familiares, características de la casa, hacinamiento, saneamiento ambiental.

La edad de las madres en ambos grupos tuvo distribución similar, observándose una mayor incidencia entre los 19 a 21 años. No se observaron diferencias significativas al comparar datos anamnésticos como estado civil, paridad, control de embarazo, estado de salud y alimentación durante el embarazo, en nuestras madres estudiadas. No hay tampoco diferencia significativas en cuanto a tipo de parto (distócico o eutócico) ni peso de las placentas. El número de fumadoras fue escaso pero similar en ambos grupos.

$\mathrm{Si}$ encontramos diferencias significativas al estudiar altura uterina en el momento del parto al comparar ambos grupos de madres (P 0,05) o que también observamos al comparar pesos y tallas de nacimiento de ambos grupos, observándose que existía relación entre la altura uterina con peso y talla de nacimiento (P 0,01). (Tabla 1).

Los niños examinados tenían edades cronológicas de 2 años 6 meses a 4 años observándose la mayor incidencia en niños de 3,7 a 4 años.

T A B L A 1

ANTECEDENTES PERINATALES

\begin{tabular}{lccc}
\hline & Control & Experimental & $P$ \\
\hline $\begin{array}{l}\text { Altura uterina de las } \\
\text { madres al momento }\end{array}$ & & & \\
$\begin{array}{l}\text { del nacimiento } \\
\% \text { normalidad }\end{array}$ & $78 \%$ & $42 \%$ & $<0,05$ \\
$\begin{array}{l}\text { Peso de nacimiento } \\
\% \text { normalidad }\end{array}$ & $100 \%$ & $0 \%$ & $<0,01$ \\
$\begin{array}{l}\text { Talla de nacimiento } \\
\% \text { normalidad }\end{array}$ & $100 \%$ & $9 \%$ & $<0,01$ \\
\hline
\end{tabular}

T A B L A 2

EXAMENES ANTROPOMETRICOS

\begin{tabular}{lrrrrc}
\hline & \multicolumn{5}{c}{ Grupo control } \\
& \multicolumn{5}{c}{ Grupo } \\
& $P 3$ & P50 & P3 & \multicolumn{1}{c}{ P50 } & $P$ \\
\hline Peso & $61 \%$ & $33 \%$ & $82 \%$ & $18 \%$ & no sig. \\
Talla de pie & $55 \%$ & $39 \%$ & $86 \%$ & $14 \%$ & $<0,02$ \\
Talla sentado & $83 \%$ & $-17 \%$ & $100 \%$ & - & $<0,02$ \\
C. craneana & $83 \%$ & $17 \%$ & $90 \%$ & $10 \%$ & no sig. \\
\hline
\end{tabular}

T A B L A 3

EXAMENES DE LABORATORIO

\begin{tabular}{lrcc}
\hline & G Control & G Experimental & $P$ \\
\hline Hematocrito & $39,84 \pm 2,59$ & $38,98 \pm 3,43$ & no sig. \\
Hemoglobina & $13,32 \pm 1,02$ & $12,91 \pm 1,4$ & $"$ \\
Calcemia & $9,43 \pm 0,42$ & $9,45 \pm 0,76$ & $"$ \\
Fosfemia & $4,26 \pm 0,73$ & $4,25 \pm 0,68$ & $"$ \\
Proteinemia & $6,63 \pm 0,67$ & $6,54 \pm 0,64$ & $"$ \\
Indice A/G & $4,02 \pm 0,42$ & $3,61 \pm 0,93$ & $"$ \\
Radiografía carpo & & & \\
\% Normalidad & $64,2 \%$ & $63,1 \%$ & " \\
\hline
\end{tabular}

Se estudió antecedentes de salud de los niños por medio de una encuesta orientada, pasada a la madre, sin que se observaran diferencias signifícativas en relación a tipo y frecuencia de enfermedades presentadas por el niño hasta el momento de esta investigación.

En relación al estudio antropométrico se observó diferencias significativas en cuanto a talla de pie $(\mathrm{P} 0,02)$ y sentado $(\mathrm{P} 0,02)$ sin que se encontraran diferencias en el estudio peso y circunferencia de cráneo. Al estudiar retardo edad ósea se observó igual porcentaje de retardo en ambos grupos (36\%). (Tabla 2).

En cuanto al examen dental practicado a los niños se observó una mayor frecuencia de hipoplasia y pigmentación del esmalte en los niños con antecedentes bajo peso $(\mathrm{P} 0,05)$.

En relación a los exámenes de laboratorio practicados no observamos diferencias significativas, llamando la atención el índice $\mathrm{A} / \mathrm{G}$ más elevado que lo normal en ambos grupos. (Tabla 3).

Al evaluar el desarrollo psicomotor de ambos grupos no se encontraron diferencias significativas observándose que más del $80 \%$ presentaba coeficiente motriz más bajo de lo normal, valores semejantes se observaron en coeficientes de lenguaje y adaptativo de los niños en estudio. Se debe hacer notar que los valores más bajos se encontraron en los niveles inferiores de la escala socioeconómica. (Tabla 4).

La encuesta alimentaria demostró que la adquisición, distribución y consumo de los alimentos no muestra diferencias significativas. 
T A B L A 4

TEST PSICOMOTRIZ

\begin{tabular}{lccc}
\hline & G Control & G Experimental & $P$ \\
\hline - Coeficiente Motriz & $16,7 \%$ & $16 \%$ & no sig. \\
$\%$ Normalidad & $11 \%$ & $9 \%$ &, \\
- Coeficiente de & $11 \%$ & & \\
$\begin{array}{l}\text { Lenguaje } \\
\% \text { Normalidad }\end{array}$ & $11,1 \%$ & $11 \%$ &, \\
- Coeficiente Adap- \\
tativo \\
$\%$ Normalidad \\
- Coeficiente Per- \\
sonalidad Social \\
$\%$ Normalidad
\end{tabular}

Conclusiones. Del análisis del trabajo se desprende que nace un número importante de niños con bajo peso y que este peso se relaciona con estrato socioeconómico, por lo que podríamos pensar que un ingreso bajo implica mala alimentación que condicionaría un aporte inadecuado de la madre al feto.

En relación al valor pronóstico que nos proporciona la altura uterina antes del parto con peso de nacimiento es importante tener en cuenta que las alturas uterinas bajas se relacionaran con pesos bajos para una misma edad gestacional y que este peso bajo se acompaña de tallas pequeñas. Parece importante recalcar este hecho porque una altura uterina insuficiente obliga una observación cuidadosa del período recién nacido, especialmente cuando se ha descrito mayor incidencia de patología en este grupo especial de niños.

En cuanto a la importancia que el peso de nacimiento tenga en el desarrollo posterior del niño de bajo estrato socioeconómico, este incidiría en la talla, observándose diferencias significativas cuando se comparan tallas de pie y sentado de niños con y $\sin$ bajo peso al nacer.

Al comparar los resultados de las pruebas psicométricas ambos grupos mostraron un retraso importante, condicionado quizás por la alta incidencia de desnutrición observada en ambos grupos, por lo que debemos hacer notar que la nutrición que recibe el niño desde su concepción más una estimulación ambiental adecuada durante sus primeros años le permitiría soportar en mejor forma las condiciones de vida cada vez más competitivas del mundo actual.

\section{SUMMARY}

47 children with low birth weight $(2.304 \pm 95)$ and 18 with normal weight at birth $(3.176 \pm 145)$, with the same gestational age (38.42 weeks) and without major clinical defccts, wcre studicd at 3 years of age using socioeconomic, anthropometric, and psychometric parameters. No signilicant, differences were found between both groups considering livings conditions, family size and jncome, cultural level and marital status of the parents, maternal age, birth order, or food habits. No differences were found upon comparing both groups with regard to diseases suffered by the children or, during their gestation, by their mothers. Both group showed a similar high incidence of malnutrition at age $3(80 \%)$. The Height of the low wcigth group was significantly lower than of the control group. Using Gesell's and Ozeretzky,s psychometric tests, no significant differences could be shown.

It is concluded that infants with low socio-economic satus are not significantly handicapped by low birth weight if they can survive to three years of age.

\section{REFERENCIAS}

1.-L. O. Lubchenco, M. D., D. T. Searle PhD J. V. Brazie M. D. The journal of Pediatrics. Vol. 81 No 4, pp. 814-822, Oct. 1972.

2.-- Erhardt. ClL. Jashi GB. Nelsen FG Krol BH and Wiener L. Amerc. J. Public. Health. 54: 1841, 1964.

3.-González, Sergio. Rev. Chil. de Pediatría, Vol, 12, pág. 1, 1969.

4.-Behrman, R. E., Balssen GS., and Lessell, R. Am. J. Dis. Child. 121: 486, 1971.

5.--Hohenahner L. Lancet: 644-645, March 27, 1971.

6.-Willermen L. and Churchill J. A. Child Develop 38: 623-9, 1967.

7.- Kaminentzky HA. et. al. American J. of Obstetrics and Gym. Vol. 115 N? 5 pp. 639-644, March 1, 1973.

8.-Sentag, L. W. and Wines, J. Amerc. y Obstet. Gynec. 54: 994, 1947.

9.-Winick, M., Rosso, P. J. Pediatrics 1969, 74, 774.

10.-Smith, C. A. Amerc. J. Obstet. Gynec. 53: 599, 1947.

11.-Roeder, L. M., Chaw, B. F. Am. Journal of Clinical Nutrition 25 Aig. 1972, pp. 812-821.

12.-Winick, P. Pediat. 74-667.

13.-Rosowski, N., Novoa F., Abarzúa y Monckeberg. Br. J. Nutrit. 25-107, 1971.

14.-Monckeberg, F. Am. J. Clin. Nutr. 25-766-72, 1972.

15.-Dobbing, John. Oxford Blackwell Scientific, Publication, 1968, pp. 287-316.

16.-Dobbing, J. Am. J. Dis. Child. 120: 411-15-1970.

17.-Dobbing J. and Jean Sands. Biol. neomate. 19: 363-78, 1972.

18.-- Givovic, Luis. Recién Nacido Desnutrido. Rev. Chil. de Ped. 10: 1015 - 1019, 1970.

19.-Gessell. Arnold. Psicología Evolutiva. Ed. Paidós 3 ed., 1960.

20.- Cochran, W. "Técnicas de Muestreo". Ed. C. E. C. S. A., México, 1971. 